\title{
Properties of a Ceramic Pyroelectric X-Ray Generator as Dependent on Residual-Gas Pressure
}

\author{
A. V. Shchagin ${ }^{a, b}$, V. I. Volkov ${ }^{b}$, V. S. Miroshnik ${ }^{b}$, A. S. Kubankin ${ }^{a}$, and A. N. Oleinik ${ }^{a, c}$ \\ ${ }^{a}$ Belgorod State University, Belgorod, 308015 Russia \\ ${ }^{b}$ National Science Center Kharkiv Institute of Physics and Technology, 61108 Kharkiv, Ukraine \\ ${ }^{c}$ John Adams Institute at Royal Holloway, University of London, Egham, United Kingdom \\ *e-mail: av.shchagin@gmail.com
}

Received July 19, 2017

\begin{abstract}
The properties of X-ray emission from a pyroelectric accelerator based on ferroelectric ceramics have been experimentally studied, including the maximum energy of X-ray photons and their maximum yield generated by accelerated electrons at various pressures of residual gas in the generator chamber. The maximum X-ray yield and maximum energy of X-ray photons and accelerated electrons have been observed at a residual-gas pressure of around several millitorr.
\end{abstract}

DOI: $10.1134 / \mathrm{S} 1063785018010194$

A compact pyroelectric accelerator was originally proposed by Brownridge [1], and its properties were subsequently studied by several research groups (see, e.g., $[2,3]$ and references therein). These investigations employed pyroelectric crystals such as lithium niobate $\left(\mathrm{LiNbO}_{3}\right)$ and lithium tantalate $\left(\mathrm{LiTaO}_{3}\right)$. When this crystal is heated or cooled in vacuum, its surface acquires an electric potential of up to $100 \mathrm{kV}$, which can be used for the acceleration of electrons or ions. Recently [4], we have proposed for the first time to use polarized ferroelectric ceramics in a pyroelectric accelerator and experimentally demonstrated the operation of a ceramic pyroelectric accelerator and Xray generator based on ferroelectric ceramics instead of a pyroelectric crystal.

This Letter presents the results of measurements of the maximum energy of photons and electrons accelerated in a ceramic pyroelectric accelerator at various residual-gas pressures. In addition, the yield of X-ray photons generated by accelerated electrons has been also studied as dependent on the residual-gas pressure. Prospects of using ceramic pyroelectric X-ray generators are discussed.

The pyroelectric X-ray generator was assembled in a cylindrical vacuum chamber that was pumped by a forevacuum pump via a liquid-nitrogen trap. The residual gas (air) pressure in the chamber was smoothly controlled by a needle valve. A cylinder made of pyroelectric ceramics was glued with a conductive epoxy composition to the end of a cylindrical copper heat sink mounted on the chamber axis. The opposite end of the heat sink was immersed into liquid nitrogen outside the chamber. The free base of the ceramic cylinder was situated in vacuum at a beryllium window of the X-ray detector. The ceramic cylinder could be resistively heated by passing current from an external source via a resistor fixed on the heat sink. The temperature was measured by a thermocouple arranged on the heat sink near the ceramic cylinder.

The $\mathrm{X}$-ray-emission spectra were measured using a spectrometer comprising an Amptek XR-100T-CdTe $(5 \times 5 \times 1 \mathrm{~mm}) \mathrm{X}$-ray detector and PX4 digital pulse processor linked to a computer. The $100-\mu \mathrm{m}$-thick beryllium window of the detector was situated in the vacuum chamber. The distance between the beryllium window and free base of the ceramic cylinder was $20 \mathrm{~mm}$. The scheme of experimental setup was presented and described in more detail previously [4].

In these experiments, we observed X-ray emission from zirconate-titanate-lead borate (TsTBS-3M) ceramics heated or cooled in vacuum chamber at residual-gas pressure. This material is traditionally used as a piezoelectric, but we employed its pyroelectric properties. The ceramic samples were manufactured in the form of $15-\mathrm{mm}$-long cylinders with a diameter of $6.4 \mathrm{~mm}$ and polarized in the axial direction. The experiments were performed with 15- and 30-mm-long samples, the latter representing two 15-mm-long cylinders glued together with a conducting epoxy composition. The ceramic cylinders were mounted in the chamber with two opposite orientations of the polarization vector so as to ensure the appearance of a negative charge on the free base on heating ("-" orientation) and positive charge on cooling ("+" orientation). These orientations are indicated by the corresponding signs in Figs. 1-3 below. 


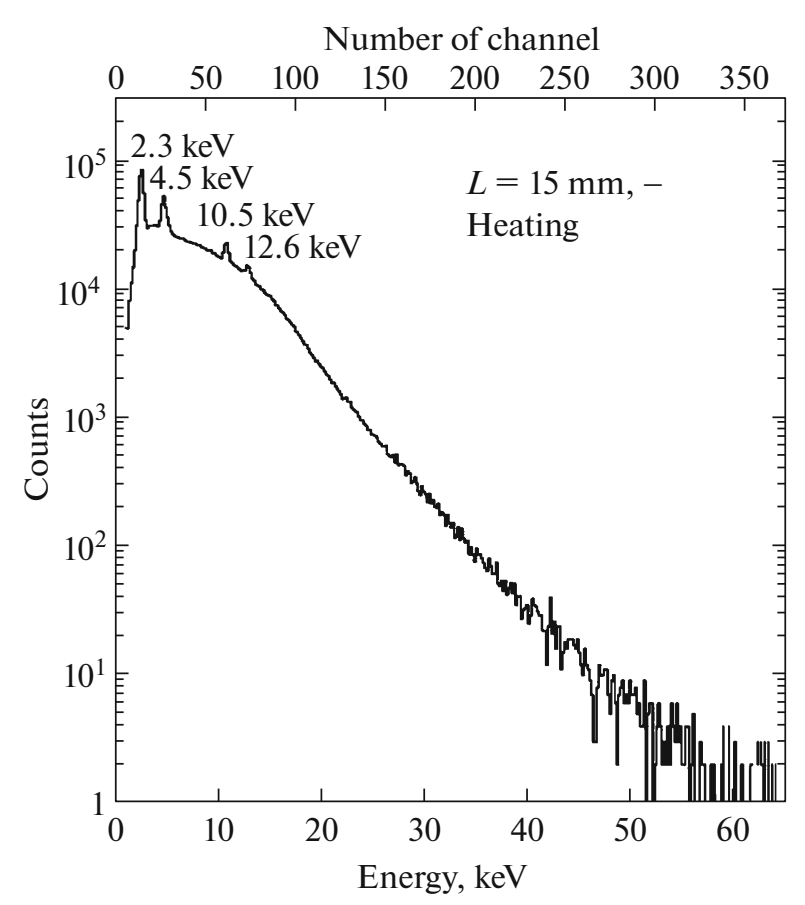

Fig. 1. X-ray-emission spectrum of a 15-mm-long ceramic sample measured during a single period of heating in the "-" orientation at a residual-gas pressure of 5 mTorr.

The X-ray-emission spectra were measured during a single period of heating or cooling of the ceramic sample. The time of heating from -50 to $+85^{\circ} \mathrm{C}$ was about $15 \mathrm{~min}$, while the duration of cooling from +85 to $-50^{\circ} \mathrm{C}$ was about $30 \mathrm{~min}$. The measurements were performed at various residual pressures for a $15-\mathrm{mm}$ long ceramic cylinder in the "-" orientation and for 30-mm-long ceramic cylinder in the "+" orientation. Figure 1 shows the typical X-ray emission spectrum measured for a 15-mm-long ceramic cylinder during heating.

Let us consider the X-ray emission from a pyroelectric cylinder with a positive charge on the free base. This charge appears when the ceramic cylinder is heated in the "-" orientation and cooled in the "+" orientation. Electrons from the residual gas are accelerated in the electric field of this charge and fall on the ceramic surface. As a result of this impact, electrons generate $\mathrm{X}$-ray bremsstrahlung radiation and characteristic $\mathrm{X}$-ray emission from atoms of ceramics. The typical spectrum presented in Fig. 1 exhibits the following characteristic X-ray peaks on a smooth background of bremsstrahlung radiation: the peak at $2.3 \mathrm{keV}$ corresponds to $L$-lines of $\mathrm{Zr}$ and $M$-lines of $\mathrm{Pb}$, the peak at $4.5 \mathrm{keV}$ corresponds to $K$-lines of $\mathrm{Ti}$ and $L$-lines of $\mathrm{Ba}$, and the peaks at 10.5 and $12.6 \mathrm{keV}$ corresponds to $L$-lines of $\mathrm{Pb}$. The characteristic peaks due to $K$-lines of $\mathrm{Zr}, \mathrm{Ba}$, and $\mathrm{Pb}$ are not observed because of limited energies of accelerated electrons and significant background of bremsstrahlung radia-
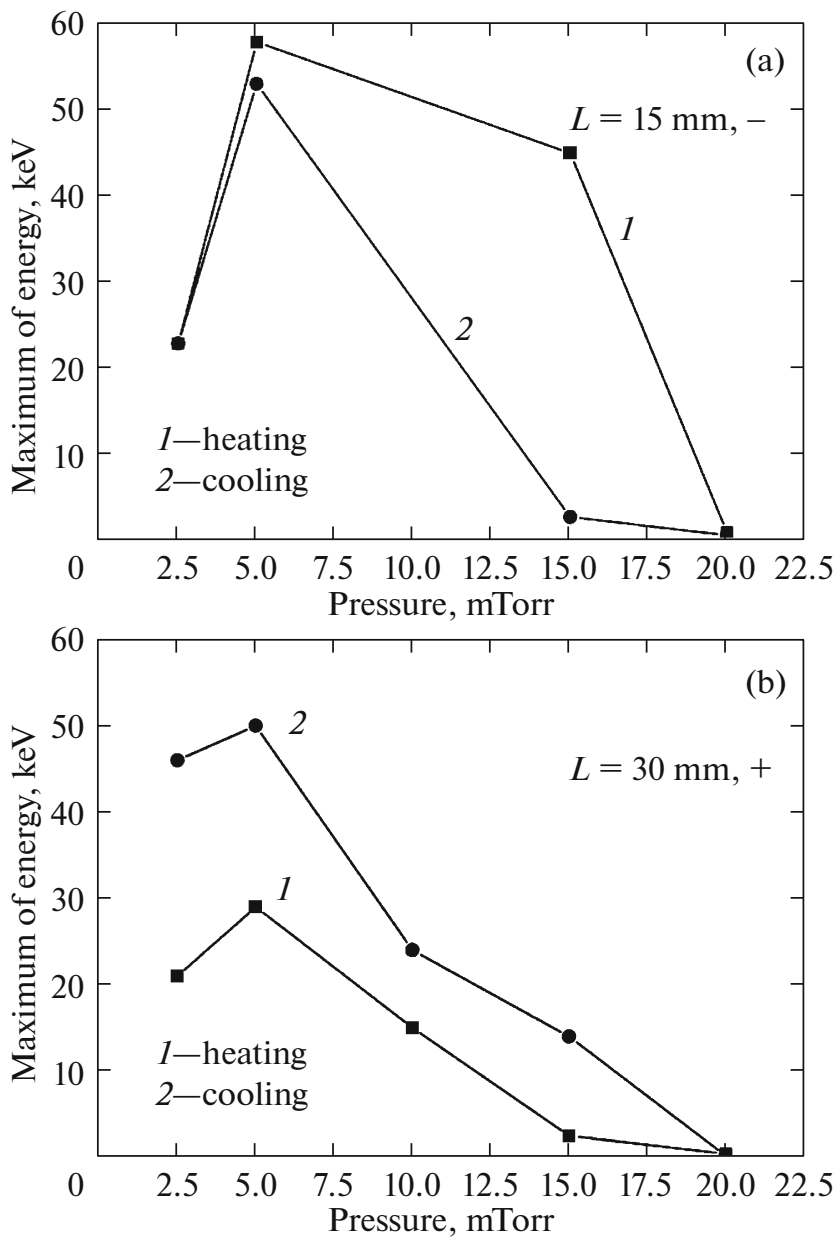

Fig. 2. Plots of the maximum energy of X-ray bremsstrahlung radiation and accelerated electrons vs. residual-gas pressure measured on heating and cooling of (a) a 15mm-long ceramic cylinder in the "-" orientation and (b) $30-\mathrm{mm}$-long ceramic cylinder in the "+" orientation.

tion. The maximum energy of bremsstrahlung radiation is about $60 \mathrm{keV}$.

Now let us consider the X-ray emission from a pyroelectric cylinder with negative charge on the free base. This charge appears when the ceramic cylinder is cooled in the "-" orientation and heated in the "+" orientation. Electrons from the surface of ceramics are accelerated in the electric field of this charge and fall into the beryllium window of the detector and onto the walls of a chamber made of stainless steel. As a result, accelerated electrons generate X-ray bremsstrahlung radiation and characteristic X-ray emission from atoms of steel walls. The resulting spectra displayed weak peaks of the characteristic radiation against a smooth background of bremsstrahlung radiation. The peaks observed at 5.4, 6.4, and $7.5 \mathrm{keV}$ were due to the $K$-lines of $\mathrm{Cr}, \mathrm{Fe}$, and $\mathrm{Ni}$ from the steel. These spectra were presented and considered in more detail elsewhere [4]. 

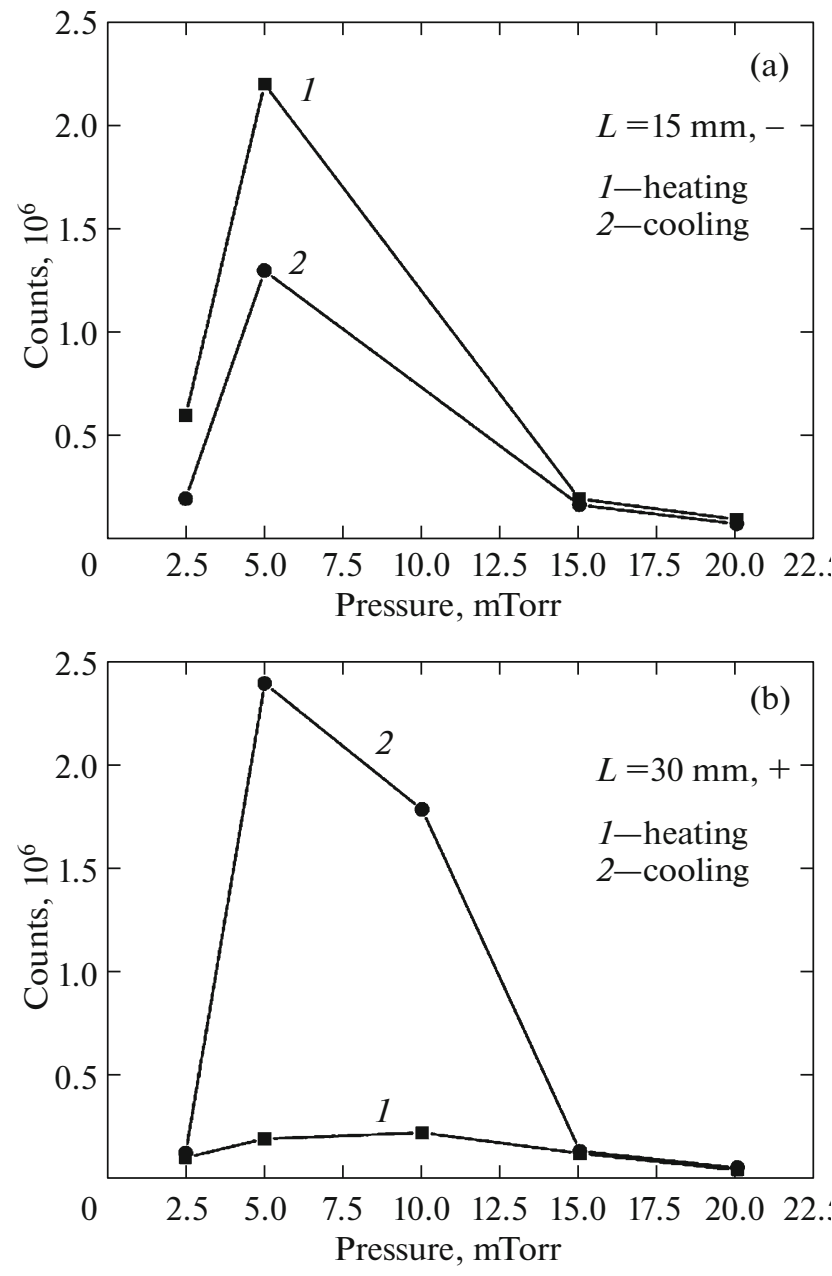

Fig. 3. Plots of the total count in the X-ray spectra vs. residual-gas pressure measured in single cycles of heating and cooling of (a) a 15-mm-long ceramic cylinder in the "-" orientation and (b) a 30-mm-long ceramic cylinder in the "+" orientation.

The maximum energy of bremsstrahlung-radiation photons observed in the spectra can be used for directly estimating the maximum energy of accelerated electrons, since the former energy cannot exceed the latter one. In the X-ray spectra measured, the maximum energy of bremsstrahlung radiation and total count significantly depended on the residual-gas pressure in the vacuum chamber of the accelerator. Results of measurements of the maximum energy of bremsstrahlung-radiation photons and maximum energy of accelerated electrons for two ceramic samples are presented in Fig. 2. Data on the total count of $\mathrm{X}$-ray photons detected by the spectrometer for the same samples are presented in Fig. 3. As can be seen from Figs. 2 and 3, the maximum energy and total count of photons exhibit no monotonic variation depending on the residual-gas pressure in the chamber. Both curves have maxima in the vicinity of 5 mTorr. These maxima are analogous to those observed in experiments with pyroelectric accelerators based on pyroelectric crystals (see, e.g., $[2,3]$ ).

The results of our experiments show that pyroelectric ceramics can be successfully used for generating $\mathrm{X}$-rays. For optimization of the use of pyroelectric ceramics in pyroelectric $\mathrm{X}$-ray generators, it is necessary to study the properties of radiation in experiments with various types of ceramics and select the optimum material. Recently, such experiments were initiated [5] with a pyroelectric generator employing lead zirconate titanate (PZT) ceramics (TsTS-19 grade). However, this ceramics showed worse results than did the TsTBS-3M composition. Evidently, pyroelectric $\mathrm{X}$-ray generators should employ ceramics with maximum ratios of the pyroelectric constant to permittivity.

Let us consider some prospects of using ceramics in pyroelectric $\mathrm{X}$-ray generators. Ceramics, in contrast to crystals, can be manufactured with an arbitrary shape and polarized in any direction. Therefore, ceramic elements of various shapes can be used in the design of a pyroelectric X-ray generator. In particular, spherical or cylindrical layers of radially polarized ceramics can be used for creating spherical and cylindrical generators, which can probably provide higher energies and currents of accelerated electrons and increased yield of $\mathrm{X}$-ray photons. Pyroelectric ceramics can also be used in the recently proposed pyroelectric undulator [6] and deflector of charged-particle beams $[6,7]$.

Acknowledgments. The work of the authors from Belgorod State University was supported in part by the Program of State Assignments in the field of scientific activities, project no. 3-1631.2017/PCh.

\section{REFERENCES}

1. J. D. Brownridge, Nature 358, 287 (1992).

2. J. D. Brownridge and S. M. Shafroth, Trends in Lasers and Electro-Optics Research, Ed. by W. T. Arkin (Nova Science, New York, 2004), p. 57.

3. V. I. Nagaichenko, V. V. Sotnikov, B. I. Ivanov, A. M. Egorov, and A. V. Shchagin, Poverkhnost', No. 3, 81 (2007).

4. A. V. Shchagin, V. S. Miroshnik, V. I. Volkov, and A. N. Oleinik, Appl. Phys. Lett. 107, 233505 (2015).

5. K. A. Vokhmyanina, O. O. Ivashchuk, V. Yu. Ionidi, A. A. Kaplii, I. A. Kishchin, A. S. Klyuev, A. S. Kubankin, M. V. Mishunin, R. M. Nazhmudinov, I. S. Nikulin, A. N. Oleinik, A. V. Sotnikov, A. S. Chepurnov, and A. V. Shchagin, Steklo Keram., No. 11, 27 (2016).

6. A. N. Oleinik, A. S. Kubankin, R. M. Nazhmudinov, K. A. Vokhmyanina, A. V. Shchagin, and P. V. Karataev, JINST 11, 08007 (2016).

7. A. S. Kubankin, A. N. Oleinik, and A. V. Shchagin, RF Patent No. $1555556716 \quad$ (2015). http://www1.fips.ru/fips_serv1/fips_servlet?DB=RUPM\&amp; DocNumber $=156716$

Translated by P. Pozdeev 